PHYSICAL REVIEW D 95, 129901(E) (2017)

\title{
Erratum: Model-independent test of general relativity: An extended post-Einsteinian framework with complete polarization content [Phys. Rev. D 86, 022004 (2012)]
}

\author{
Katerina Chatziioannou, Nicolás Yunes, and Neil Cornish \\ (Received 12 May 2017; published 6 June 2017)
}

DOI: 10.1103/PhysRevD.95.129901

In this erratum, we correct a mistake in the deconstruction of the inspiral waveform generation in the parametrized postEinsteinian framework. These corrections do not affect the conclusions of the paper.

In the paper, we described how to construct a gravitational waveform due to the inspiral of two compact objects in General Relativity and in the parametrized post-Einsteinian (ppE) framework. We recently discovered a few mistakes in Sec. IV of the paper that we correct here. None of these corrections affects the conclusions of our work, but they do affect some of the intermediate expressions, as we describe below.

We begin through the ppE correction to the reduced effective potential,

$$
V_{\mathrm{eff}}=\left(-\frac{m}{r}+\frac{L_{z}^{2}}{2 r^{2}}\right)\left[1+A\left(\frac{M}{r}\right)^{p}\right]
$$

where $m$ is the total mass of the system, $r$ is the orbital separation, $L_{z}$ is the $\mathrm{z}$ component of the angular momentum, and $A$ and $p$ are $\mathrm{ppE}$ amplitude and exponent modifications. Taking the radial derivative of this effective potential and setting it to zero, we find the modified Kepler's law, as given in Eq. (91) of the paper. Since the effective potential is, in this case, equal to the binding energy (since the radial kinetic energy term is zero for circular orbits), we find

$$
E_{b}=-\frac{1}{2} \eta^{-2 / 5}(2 \pi \mathcal{M} F)^{2 / 3}\left[1-\frac{1}{3} A(2 p-3) \eta^{-2 p / 5}(2 \pi \mathcal{M} F)^{2 p / 3}\right]
$$

where $\mathcal{M}$ is the chirp mass, $F$ is the orbital frequency, and $\eta$ is the symmetric mass ratio. This expression corrects Eq. (94) in the paper.

From the corrected expression for the binding energy presented above, we can now compute the rate of change of the orbital frequency with time,

$$
\frac{d F}{d t}=\frac{48}{5 \pi \mathcal{M}^{2}}(2 \pi \mathcal{M} F)^{11 / 3}\left[1+B \eta^{-2 q / 5}(2 \pi \mathcal{M} F)^{2 q / 3}+\frac{1}{3} A\left(2 p^{2}-2 p-3\right) \eta^{-2 p / 5}(2 \pi \mathcal{M} F)^{2 p / 3}\right],
$$

which corrects Eq. (98) in the paper. Notice that there was an inconsequential typographical error in the inline equation between Eqs. (51) and (52), where the binding energy was missing a factor of $1 / 2$. Notice also that in deriving $d F / d t$ above we used the rate of change of the binding energy (i.e., the energy flux), as written in Eq. (96). When writing this flux as a function of the orbital frequency, we implicitly assumed that $\dot{E}_{\mathrm{GR}}$ is proportional to $v^{2}(\mathrm{~m} / r)^{4}$. If one assumes a different form for the General Relativity expression, such as one that is proportional to $r^{4} \omega^{6}$, one will find slightly different expressions for the waveform due to the $\mathrm{ppE}$ modifications to Kepler's third law.

Propagating these corrections further, we encounter the Fourier transform of the ( $\ell$ harmonic of the) gravitational waveform. The latter is defined in Eqs. (17) and (18) of the paper, which have an inconsequential typographical error since both expressions should depend on the second derivative of the quadrupole moment $\ddot{Q}_{i j}$ instead of $Q_{i j}$. The corrected Fourier transform of the waveform is

$$
\tilde{h}^{(\ell)}=\sqrt{\frac{5 \pi}{48 \ell}} Q \frac{\mathcal{M}^{2}}{D} \eta^{(2-\ell) / 5} u_{\ell}^{(2 \ell-11) / 2} e^{-i \Psi^{(\ell)}}\left[1-\frac{1}{2} B \eta^{-2 q / 5} u_{\ell}^{2 q}+\frac{1}{6} A\left(\ell p+3+2 p-2 p^{2}\right) \eta^{-2 p / 5} u_{\ell}^{2 p}\right],
$$

correcting Eq. (99) in the paper. Here, $Q$ is a function of some sky angles, $u_{\ell}$ is a reduced frequency, $D$ is the distance to the source, and $B$ and $q$ are ppE amplitude and exponent corrections to the energy flux (all of which are defined in more detail in the paper). Similarly, the corrected phase of the Fourier transform is 


$$
\delta \Psi^{(\ell)}=\frac{5}{64} A \frac{\ell\left(2 p^{2}-2 p-3\right)}{(4-p)(5-2 p)} \eta^{-2 p / 5} u_{\ell}^{2 p-5}+\frac{15}{64} B \frac{\ell}{(4-q)(5-2 q)} \eta^{-2 q / 5} u_{\ell}^{2 q-5},
$$

which corrects Eq. (100) in the paper. Finally, the correction to the Fourier phase at the $p=4$ pole remains equal to Eq. (100) in the paper but with the prefactor 280/9 instead of 880/9, while that at $p=5 / 2$ remains equal to Eq. (103) in the paper but with the prefactor $20 / 3$ instead of 30 .

These modifications to the inspiral gravitational waveform then lead to modifications to the mapping between ppE coefficients and ppE modifications to the binding energy. In particular, Eqs. (106) and (107) in the paper are now corrected to

$$
\begin{aligned}
& \alpha=\left[\frac{1}{6} A\left(3+4 k-2 k^{2}\right)-\frac{1}{2} B\right] \eta^{-2 k / 5}, \\
& \beta=-\frac{5}{64} \frac{1}{(4-k)(5-2 k)}\left[A\left(2 k^{2}-2 k-3\right)+3 B\right] \eta^{-2 k / 5},
\end{aligned}
$$

and Eqs. (109) and (110) in the paper are now corrected to

$$
\begin{aligned}
& \alpha=\frac{1}{6} A\left(3+4 p-2 p^{2}\right) \eta^{-2 p / 5}, \\
& \beta=-\frac{5}{64} \frac{1}{(4-p)(5-2 p)} A\left(2 p^{2}-2 p-3\right) \eta^{-2 p / 5} .
\end{aligned}
$$

Similarly, Eq. (118) in the paper becomes

$$
c_{\mathrm{cons}}=-\frac{8}{15} \frac{b(3-b)\left(b^{2}+6 b-1\right)}{b^{2}+8 b+9},
$$

and Eq. (120) in the paper becomes

$$
c_{\text {both }}=-\frac{16}{15} \frac{b(3-b)\left(b^{2}+7 b+4\right)}{b^{2}+8 b+9} .
$$

